\title{
Présentation. L'exercice de rhétorique comme étude du cœur et de l'esprit humain et comme édification de soi
}

\section{Jean-Yves Vialleton}

\section{(2) OpenEdition \\ Journals}

\section{Édition électronique}

URL : http://journals.openedition.org/rhetorique/192

DOI : $10.4000 /$ rhetorique. 192

ISSN : 2270-6909

Éditeur

UGA Éditions/Université Grenoble Alpes

Édition imprimée

ISBN : 978-2-84310-263-9

\section{Référence électronique}

Jean-Yves Vialleton, «Présentation. L'exercice de rhétorique comme étude du cœur et de l'esprit humain et comme édification de soi », Exercices de rhétorique [En ligne], 2 | 2013, mis en ligne le 18 novembre 2013, consulté le 12 septembre 2020. URL : http://journals.openedition.org/rhetorique/192 ; DOI : https://doi.org/10.4000/rhetorique.192

Ce document a été généré automatiquement le 12 septembre 2020.

\section{c) (i) (2)}

Les contenus de la revue Exercices de rhétorique sont mis à disposition selon les termes de la Licence Creative Commons Attribution - Pas d'Utilisation Commerciale - Partage dans les Mêmes Conditions 4.0 International. 


\section{Présentation. L'exercice de rhétorique comme étude du cœur et de l'esprit humain et comme édification de soi}

Jean-Yves Vialleton

1 L'Essai de rhétorique dans la traduction de quatre harangues de Tite Live, avec des notes par M. de Crousaz ${ }^{1}$ est le " chef d'œuvre » (au sens que le mot a dans l'artisanat) d'un professeur de rhétorique et un échantillon de la nouvelle pédagogie qu'il essaie de promouvoir. C'est, selon les mots de l'auteur même, un "petit volume " donnant l'« essai d'une méthode différente » pour enseigner l'éloquence ${ }^{2}$. Son auteur, Jean-Pierre de Crousaz (1663-1750), le publie en 1725, à Groningue, alors qu'il enseigne dans l'université de cette ville, après avoir dû quitter l'Académie de Lausanne à cause d'une querelle religieuse. Le livre est dédié au gouverneur du prince d'Orange (qui d'après l'épître dédicatoire le lui aurait demandé). L'année qui suit la publication, Crousaz est choisi comme gouverneur du prince héritier d'une cour allemande (Frédéric de Hesse-Cassel).

2 Il est difficile au lecteur d'aujourd'hui de comprendre ce qui faisait le grand intérêt de ce court livre (in $8^{\circ}, 176 \mathrm{p}$.) : le commentaire qu'il contient peut n'apparaître à première lecture que comme une paraphrase bien peu instructive. Mais en réalité, il s'agit d'une analyse rhétorique tout à fait technique et qui plus est fondée sur une conception réfléchie de ce qu'est la rhétorique et de ce à quoi elle peut servir dans l'éducation. C'est ce qu'on espère montrer ici.

\section{Principes d'édition}

Le livre porte sur quatre discours tirés de l'Histoire romaine, qui forment deux paires en pour et en contre. Nous éditons le premier des quatre.

Le premier couple de discours concerne une négociation en temps de guerre, peu avant la bataille de Zama (202 av. J-C.). Lors de la seconde guerre punique, Hannibal qui était 
prêt de marcher sur Rome connaît des revers et il est en outre rappelé à Carthage attaquée par Rome. Scipion a en effet débarqué en Afrique, et s'approche de Carthage même : Scipion, qui sera baptisé «l'Africain ", est dans l'histoire romaine l'équivalent de Bonaparte dans l'histoire française, un très jeune général qui enthousiasme ses troupes et va de victoire en victoire. Hannibal essaie de négocier avec Scipion, mais la négociation échoue et il est vaincu à Zama, ce qui signe la fin de sa longue et brillante carrière militaire. Tite-Live (XXX, 30-31) donne et le discours d'Hannibal et la réponse de Scipion, et Crousaz commente les deux.

Le deuxième couple de discours constitue un débat pour ou contre l'abolition d'une loi, la loi Oppia, loi somptuaire sur la tenue des femmes (184 av. J.-C.). Caton l'Ancien (ou le Censeur) demande son maintien, le tribun de la plèbe Valère lui répond et triomphe de ses arguments (Tite-Live, XXXIV, 2-4 et 5-7) ${ }^{3}$.

Pour chaque couple de discours, Crousaz donne d'abord le texte latin et une traduction en regard, puis des "remarques ", c'est-à-dire un commentaire au fil du texte, dans lequel est redonné, morceau par morceau, le texte latin et sa traduction (parfois un peu modifiée).

7 Nous reproduisons donc ici les seules «Remarques sur le discours d'Hannibal à Scipion ». Pour le texte français, nous avons modernisé l'orthographe (mais conservé la casse et la ponctuation), et inséré entre crochets les numéros de page de l'édition originale (p. 26-62). Pour le texte latin de Tite-Live, nous suivons de même l'usage actuel (pas d'accents, $i$ et non $j$, $u$ et non $v$, ae et non $\propto$, oe et non $\propto$, etc.), tout en respectant la casse et la ponctuation de Crousaz; nous ajoutons entre crochets les références au découpage traditionnel en paragraphes (\$3-30), découpage apparemment postérieur (il daterait de $1738^{4}$ ) et qui ne correspond pas toujours avec celui de Crousaz. Enfin, nous ne signalons pas les différences entre le texte latin que donne Crousaz, ici reproduit, et celui des éditions actuelles (par exemple, omission de sorte au $\S 3$, etc.).

8 Nos notes de bas de page visent à montrer le caractère technique du commentaire rhétorique et la manière dont il s'inscrit dans la tradition ${ }^{5}$ : références à la Rhétorique d'Aristote (abrégé Aristote) et à d'autres traités classiques (Rhétorique à Herennius, abrégé Her. ; Institution oratoire, abrégé Quintilien). Elles visent aussi à dégager la spécificité de l'approche de Crousaz. Pour cela, elles donnent des renvois aux analyses d'autres professeurs de rhétorique, qu'ils soient auteurs d'amples commentaires de Tite-Live, Lorich $\left(1537^{\circ}\right)$, Tesmar $\left(1657^{7}\right)$, Ferrazzi $\left(1694^{8}\right)$, ou compositeurs de conciones ${ }^{9}$, Botidoux $\left(1823^{10}\right)$, Vauchelle $\left(1881^{11}\right)$.

9 Nous avons ajouté des subdivisions dans le commentaire de Crousaz, qui ne correspondent pas au plan du commentaire lui-même, mais aux divisions du texte commenté, telles que le commentaire les suggère. Ce que nous proposons là est donc une hypothèse de division, justifiée dans les notes du texte (notes 30, 33, 57, 61, 76 et 86). Sur la base de cette reconstitution, nous avons inséré dans le fil du commentaire des lettres entre crochets (accompagnées, pour les grandes parties, d'un intertitre) de façon à marquer les parties du discours d'Hannibal (de $A$ à $D)$ et ses sous-parties ( $A a, A b$, etc.). 


\section{L'œuvre de Crousaz} un recueil de sermons, des ouvrages sur l'éducation et de nombreux ouvrages de philosophie dans l'acception que le mot avait dans le système éducatif de l'époque, c'est-à-dire philosophie et théologie, mais aussi logique, morale, physique (au sens large de l'époque: étude de la Nature, ce qui inclut la mécanique et la biologie), mathématiques. Son œuvre majeure est une logique (Système de réflexions qui peuvent contribuer à la netteté et à l'étendue de nos connaissances, ou Nouvel essai de logique, 1712). Le livre connaît de nombreux avatars : version latine, version abrégée (« avec une préface sur l'usage et l'abus des abrégés »), différentes versions complétées, qui en font moins un traité de logique qu'un ample ouvrage de philosophie morale (six volumes pour la quatrième édition, Lausanne et Genève, Marc-Michel Bousquet et compagnie, 1741).

11

Crousaz n'est connu aujourd'hui que pour son Essai sur le beau, 1715, cité et discuté par Diderot (réédité chez Fayard, « Corpus des œuvres de philosophie en langue française », 1985). Sur le reste de son œuvre, la bibliographie est maigre. Par exemple, dans le répertoire bibliographique annuel de la Revue d'Histoire Littéraire de la France entre 1986 et 2008, un seul article est indiqué : c'est un article de musicologie sur le passage d'esthétique musical, ajouté dans les rééditions de l'Essai sur le beau. On trouve des mentions rapides dans quelques livres d'histoire de l'éducation. Heureusement, Crousaz a fait l'objet d'une monographie : Jacqueline de La Harpe, Jean-Pierre de Crousaz et le conflit des idées au siècle des Lumières, Genève, Droz, 1955. Ce livre rappelle notamment les difficultés que ce protestant éclairé rencontra face à ses coreligionnaires de Lausanne (querelle du Consensus, formulaire imposé au clergé et aux enseignants par Berne contre les novateurs). On y trouve des bibliographies primaire et secondaire. Crousaz ne semblait guère devoir retenir l'attention que dans sa patrie (voir Daniel Christoff, La Philosophie dans la Haute École de Lausanne : 1542-1955, Lausanne, Université de Lausanne, 1987), où est d'ailleurs conservé un fonds le concernant ${ }^{13}$. On sent pourtant depuis peu les signes d'une redécouverte de son œuvre. Un Congrès international des Lumières à Los Angeles en 2003 lui a consacré une journée. Un numéro de la Revue de théologie et de philosophie lui a été consacré en 2004 ( $n^{\circ}$ 136). Les travaux de ce début de XXI ${ }^{e}$ siècle semblent donner raison à Daniel Mornet. Dans sa préface au livre de J. de La Harpe, celui-ci affirmait que Crousaz, dont les correspondants étaient parfois illustres (Fontenelle) et dont la participation aux débats scientifiques et théologiques de l'époque était active (textes polémiques contre Bayle, Leibniz, Wolf...), était une personnalité injustement méconnue de l'Europe des Lumières.

Plusieurs des écrits de Crousaz, qu'il publie en Hollande, concernent la pédagogie et contiennent des passages sur l'apprentissage du latin et sur l'éloquence, qui éclairent l' Essai de rhétorique: Nouvelles maximes sur l'éducation des enfants $\left(1718^{14}\right)$, ouvrage à la Swift, entièrement écrit par antiphrase et rempli de traits polémiques et d'allusions biographiques, à la défense des «anti-conformistes » (veine polémique qu'on retrouve dans la conclusion des Essais); Traité de l'éducation des enfants, "destiné aux précepteurs », $1721^{15}$; «Discours libres sur l'Instruction du bas Collège » (ou «Pensées libres sur les instructions publiques »), « De l'instruction publique dans les auditoires » (c'est-à-dire les Académies, les lycées protestants), "Discours de la pédanterie », rassemblés dans le tome II des Divers ouvrages de monsieur de Crousaz..., 17376. Par 
ailleurs, la lecture de sa Logique, qui est d'abord l'ouvrage d'un moraliste, éclaire grandement sa conception de la rhétorique.

\section{Crousaz et la rhétorique}

13 Dans les textes latins, comme Tite-Live dans l'Essai..., ce n'est pas le latin qui intéresse Crousaz. Pour lui, la connaissance du latin ne doit pas être un but en soi ${ }^{17}$, mais le moyen d'accéder à des textes qui forment le "goût ", les " mœurs » et la " politesse » de l'élève et donc sa capacité à "bien parler ». L'usage des bonnes traductions en français ou en anglais des auteurs grecs et latins, loin d'être néfaste, permet une comparaison instructive entre les langues, analogue à l'anatomie comparée en biologie. De toute manière, comme le pensait Descartes et comme le montre la Grammaire de Port-Royal, à toutes les langues peuvent s'appliquer de mêmes « Maximes, ce qui fait voir que règne en chacune beaucoup plus de Raison que de Bizarrerie ${ }^{18} »$. rhétorique, bien au contraire. Celle-ci reste la clé des études littéraires : «Pour profiter davantage de la lecture d'Homère, de Virgile et en général des Poètes, il est important que des jeunes gens fassent cette étude sous la direction d'un Précepteur qui ait lu avec attention la Rhétorique et la Poétique d'Aristote ${ }^{19}$ ». Ce qu'il cherche à moderniser, et même à " civiliser ", comme disait Guez de Balzac au siècle précédent, c'est la méthode.

D'abord, il propose non un traité, mais un commentaire : il croit, nous dit le Journal des savants qui rend compte de l'Essai, «que les préceptes de la Rhétorique ne peuvent pas mener fort loin et que les exemples servent plus que les règles pour former les Orateurs ${ }^{20} »$.

16 Son commentaire de Tite-Live est entièrement rédigé, dans un style simple et parfois même oral, sans notions exprimées en latin ou en grec, sans même de calque du grec (si ce n'est le mot antithèse), sans cette "pédanterie » que Crousaz détestait ${ }^{21}$, enfin de façon vivante (utilisation du style indirect libre). Pour l'analyse de la manière de mener le raisonnement, il refuse les analyses visant à rendre compte de la logique du texte en explicitant les syllogismes sur lequel le discours repose, comme on le voit chez Ferrazzi (ce qu'il appelle les analyses logiques à la façon de Ramus ${ }^{22}$ ). Il refuse comme stérile (dans la lignée de la Logique de Port-Royal ${ }^{23}$ ) l'apprentissage mécanique des "lieux ${ }^{24}$ " (des catégories par lesquelles sont trouvés les arguments), préférant les faire remarquer au fil de la lecture d'un texte, comme on le voit dans ces commentaires. Il ne relève pas les «maximes » en fin d'analyse, mais les marque (en capitales) dans le fil du commentaire. C'est qu'il ne faut pas selon lui faire apprendre des sentences et des citations à l'élève, mais lui faire apprendre par cœur l'ensemble du discours ${ }^{25}$. Pour la composition, il préfère procéder par glissements progressifs que livrer la désignation claire des différentes parties, de façon à souligner l'unité organique du discours, sa " conduite ", faite de "répétitions » propres à "imprimer dans l'esprit» un point

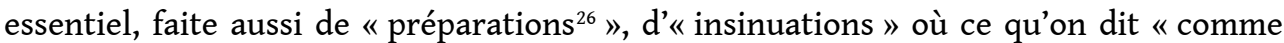
en passant » compte autant que les « raisons » mises en avant. Crousaz reprend à la grammaire de Port-Royal la notion d'« idées accessoires ».

L'exemple de son analyse de l'exorde suffira ici à fixer sa manière. Pour le discours d'Hannibal, les commentateurs mentionnés plus haut s'accordent à voir un exorde qui comporte les trois premières phrases, en finissant aux mots finem imposuisse. Crousaz parle lui aussi d'exorde, mais à propos de la seule première phrase, et il n'en marque 
pas la fin. Mais on peut supposer qu'il adopte le même découpage, puisqu'il voit dans la troisième phrase des "ménagements", une façon de "prévenir les sentiments" défavorables que Scipion pourrait avoir à l'évocation de son père juste avant. De plus, il marque une rupture dans la séquence suivante : «Jusqu'ici la personne de Scipion est entrée dans tout ce qu'Hannibal vient de dire; mais il quitte cette idée pour passer à des maximes générales ». On a donc la fin d'un exorde "a persona auditoris ", qui tire argument de la personne de l'auditeur. Mais la suite évoque encore la manière de "disposer celui qui écoute", d'« engager" "à une attention nouvelle", comme si l'exorde n'était pas fini. À l'inverse, l'exorde anticipe sur le corps du discours, puisqu'Hannibal y va déjà « adroitement à son principal but », suscitant une crainte qui fera préférer la paix.

La conception de la rhétorique qui est celle de Crousaz est explicitée dans le second paragraphe, qui pourtant ne semble qu'un rappel banal. Il y souligne qu'est nécessaire d'« alléguer des raisons", de suivre un " ordre ", de choisir les " expressions » : c'est l'invention, la disposition et l'élocution (« pisteis », «lexis », « protaxis », Aristote, III, 1, 1403 b 6-15), c'est-à-dire les moyens de persuasion par le logos. Mais, dit-il, « cela ne suffit pas ». Il insiste alors sur les autres moyens de persuasion, énoncés par Aristote au début de sa Rhétorique (I, 2, 56a1 sq.): le caractère de l'orateur («èthos)» et la disposition de l'auditeur (" diatheinai pôs »). Cette insistance marque un parti pris dans un débat aussi vieux que la rhétorique et qui apparait dans l'hésitation d'Aristote luimême : le premier chapitre du livre I de la Rhétorique insiste sur l'analyse du logos et la donne comme une étude jamais faite jusqu'alors, mais toute la suite donne beaucoup d'importance aux autres moyens, ceux qu'avaient mis en avant les prédécesseurs d'Aristote.

19 Ce qui semble donc intéresser Crousaz, ce n'est pas la structure formelle des raisonnements, mais ce qui dans la rhétorique relève de la connaissance de l'esprit et du cœur. Nous dirions, en termes modernes, de la psychologie, psychologie cognitive à caractère universelle ou psychologie morale propre à chaque caractère ou chaque mentalité. Par là, il redonne toute sa place au contenu moral du livre II de la Rhétorique d'Aristote (pathos et èthos, ou en latin motus et mores), qu'on est tenté de considérer comme une digression. Il propose en cela une rhétorique bien différente de ce que nous appelons aujourd'hui étude de l'argumentation, et plus riche. Les lois cognitives et psychologiques sont parfois explicitées, en particulier dans les discours sur la loi Oppia, et parfois reprises d'Aristote: "Nos erreurs consistent ordinairement en ce que nous confondons des choses différentes; on s'en dégage en distinguant "; "L'esprit n'aime pas s'en occuper [des idées tristes]; il leur refuse son attention, pour le tourner vers des objets plus satisfaisants » (discours de Valère contre la loi Oppia). Ces lois peuvent déboucher sur une description des vertus qui rappellent l'écriture des moralistes : « Il est assez naturel aux hommes à s'évaporer en réflexions sur le passé ; c'est même là une consolation fort ordinaire aux misérables; mais c'est une consolation des plus faibles, et des plus inutiles. Mais ceux qui ont la prudence au lieu de se borner à des souhaits infructueux, et à regretter le passé sur lequel ils ne peuvent rien, en profitent pour mieux régler l'avenir » (discours d'Hannibal).

La rhétorique se fonde donc sur la connaissance des hommes, et en cela les grands discours sont moraux. Mais ils le sont aussi en ce qu'ils donnent une peinture de l'orateur, en tant qu' « oratio morata». Le commentaire des discours sur l'abrogation de la loi Oppia commence et finit par des portraits moraux des deux orateurs. Pour le 
discours d'Hannibal, Crousaz donne à la fois les motivations véritables du général carthaginois, les airs que celui-ci se donne dans le discours (son ethos rhétorique) et ce que son discours dit malgré lui sur celui qui le prononce. Dans la dissociation de ces deux derniers points réside l'échec du discours. Hannibal a peur, et avec raison : « Il craint qu'une longue suite de malheurs n'ait fait perdre à ses troupes celle [la confiance] qu'elles avaient en lui. Il considère que l'armée de Scipion ne peut avoir d'espérance qu'en la victoire, au lieu que ses Soldats ont les murs de Carthage pour retraite, et savent que la fuite les peut garantir de la mort. Toutes ces raisons lui font souhaiter la paix.» Mais ce sont d'autres personnages qu'il joue face à Scipion, tellement plus jeune que lui : l'ami, le père, l'homme d'expérience, le sage à l'esprit tranquille. Et c'est sa vraie personnalité qui apparaît pourtant: «Le long discours d'Hannibal marque sa crainte. Il s'efforce de faire comprendre à Scipion que c'est pour lui qu'il craint, et que cette crainte est l'effet de son habileté même et de son expérience. Le peu de paroles de Scipion [dans sa réponse à Hannibal] marque son courage et ses espérances, fondées aussi sur ses lumières. " Comme au théâtre, la rhétorique ratée est parfois plus instructive que la bonne du point de vue de la connaissance morale.

Bien parler, c'est connaitre l'homme et se connaître soi-même. C'est sur la même connaissance que se fonde le bon enseignement selon Crousaz. Les quatre discours choisis ne l'ont pas seulement été pour leur célébrité. Le couple que constitue un discours et sa réponse forme un débat pareil à un match sportif et, dans les deux débats choisis, comme dans les contes, c'est le petit qui gagne contre le grand, le jeune contre le vieux, scénario propre à "capter l'attention » d'un jeune élève : quand Crousaz, à soixante trois ans, prend en main l'éducation de Frédéric de Hesse-Cassel, l'enfant n'a que six ans.

En même temps s'«impriment» dans l'esprit de l'élève deux "imagines", deux exemples édifiants. Le premier vainqueur, Scipion, figure un jeune héros sage, qui, tout en restant plein de dignité et de pudeur, sait éviter avec fermeté les pièges qu'on lui tend, y compris ceux de la "politesse" (Crousaz présente Scipion face à Hannibal plutôt comme un homme bien élevé que comme un négociateur plein de haine et en position d'être inflexible). Le second vainqueur, face à l'austère Caton, est le bien nommé Valère. Ce héros illustre l'humour et la galanterie moderne (la fréquentation des femmes n'est pas inutile) qui réussissent à s'opposer à la vénérable sévérité des temps anciens et montrent que douceur vaut mieux que force ni que rage. Et tout cela se fait, non en latin, ni même en néerlandais ou en allemand, mais en français. Ainsi l'exercice rhétorique permet une éducation complète, où compte d'abord la nécessité $d^{\prime}$ '« animer aux bonnes mœurs et aux manières aimables » les jeunes élèves ${ }^{27}$.

\section{NOTES}

1. Groningue, J. Sipkes, 1725, cahiers A-L, 176 p. (BnF Tolbiac, X- 17775 et Google Livres) : premier cahier signé* (épître dédicatoire et préface au lecteur), puis étude de quatre discours tirés de Tite-Live, enfin, p.172, violent texte polémique contre un livre de rhétorique du théologien 
catholique Matthäus Tympe (Tympius, mort après 1615), Dormi secure vel Cynosura professorum ac studiosorum eloquentiae, seu centum et viginti themata oratoria..., Cologne, P. von Brachel, 1613 (Google a la rééd. 1615).

2. Ces mots de la préface de l'Essai seront repris par Crousaz dans son discours « Des instructions publiques et des auditoires » (Divers ouvrages de monsieur de Crousaz, Amsterdam, aux dépens de la Compagnie, 1737, t. II, dans la section « Du professeur en éloquence ", où est repris l'exemple de la harangue du jeune Scipion : long passage sur le rôle du professeur p. 101-166; mention de l' Essai, p. 114 ; harangue de Scipion, p. 129 sq.).

3. Pour le commentaire des discours sur la loi Oppia et sa comparaison avec d'autres commentaires, voir F. Goyet, "Les analyses de discours dans le livre XIII des Eloquentiae sacrae et humanae parallela", dans Sophie Conte éd., Nicolas Caussin : rhétorique et spiritualité à l'époque de Louis XIII, Marburg, LIT Verlag (collection Ars rhetorica), 2006, p. 1-48 (Actes du colloque Caussin, Troyes, 2004).

4. Selon R. S. Conway cité par J. Bayet et G. Baillet (introd. à Tite-Live, Histoire romaine, tome I, Paris, Les Belles Lettres, 1965, p. CXXVII), ce paragraphage est sans doute dû à Drakenborch, en 1738 : car on ne le trouve pas dans l'édition, légèrement antérieure, de Crevier.

5. Elles apportent par là aussi une minuscule contribution à un grand travail possible de répertoire $\mathrm{du}$ vocabulaire rhétorique français et de sa concordance avec le vocabulaire latin et néo-latin, travail esquissé par le lexique que donne Heinrich Lausberg au $\$ 1246$ de son Handbuch (p. 845-921 de la trad. anglaise, Handbook of Literary Rhetoric, Leyde, Brill, 1998).

6. T. Livi patavini orationes omnes, ex libris de II. bello Punico, Artificio Dialectico et Rhetorico illustratce... Francfort, Christianus Egenolphus, discours 44, 149v ${ }^{\circ}-156 \mathrm{r}^{\circ}$ (bsb10996146, images 324-337).

7. Rhetoricarum exercitationum libri VIII, Amsterdam, Elzévir (éd. posthume par Daniel de Stephani), livre V : Exercitationum rhetoricarum liber V qui est Commentariolus Analyticus in orationes T. Livii, quotquot earum supersunt, hoc est, ducentas quinque..., discours 145, p. 425-427 (Google).

8. Exercitationes rhetoricae in orationes T. Livii, Padoue, Presses du Séminaire [Giovanni Manfrè], discours 118, p. 207-211 de l'éd. 1740 qu'ont Gallica et Google.

9. Les conciones sont des recueils scolaires de discours choisis. Ils se situent dans la tradition d'Henri Estienne qui a publié en 1570 un recueil de grands discours de l'Antiquité tirés des historiens grecs et latins (Conciones : sive Orationes ex graecis latinisque historicis excerptae...). Il existe sur ce modèle des recueils ajoutant des "harangues" modernes aux discours antiques (par exemple Harengues militaires, et concions de princes, capitaines, embassadeurs, et autres manians tant la guerre que les affaires d'Estat..., Paris, Nicolas Chesneau, 1572, trad. par Fr. de Belleforest des Orationi militari de Remigio Nannini, 1560). La tradition de l'étude rhétorique des grands discours «historiques" ne s'éteint en France qu'à la toute fin du XIX siècle (voir A.Compagnon, La Troisième République des Lettres, Paris, Le Seuil, 1983).

10. Jean Le Deist de Botidoux, Conciones et discours choisis dans Salluste, Tite-Live, Tacite et QuinteCurce, Paris, Libr. classique et d'éducation, Veuve Maire-Noyon, A. Pigoreau, successeur, p. 492-499. - Nous remercions Anne Vibert d'avoir signalé et procuré cet ouvrage à l'équipe RARE.

11. Cette première éd. (Paris, Poussielgue Frères) n'est pas à la BnF. Édition utilisée : Abbé Alphonse-Arthur Vauchelle, dit au titre «supérieur du petit séminaire de Noyon ", Conciones latinae e sacris necnon e profanis scriptoribus excerptae. Nouveau recueil, publié avec exposés historiques, plans et notes en français, Paris, Librairie Ch. Poussielgue, 1898, $3^{\mathrm{e}}$ éd., discours 27, p. 170. Autres éditions à la BnF: même éditeur, 1892, 2éd., et J. de Gigord, 1912, $2^{\mathrm{e}}$ éd. [sic]. Chez ce dernier éditeur existe une autre édition datée 1923.

12. Voir Alexandre Cioranescu, Bibliographie de la littérature française du XVIII siècle, Genève, Slatkine Reprints, 1999, n²1906-21935 et n² 21940.

13. Au Département des manuscrits de la Bibliothèque cantonale et universitaire : voir Marianne Perrenoud, Inventaire des Archives Jean-Pierre de Crousaz, 1663-1750, Lausanne, Bibliothèque cantonale et universitaire, 1969. 
14. Amsterdam, L'Honoré et Chatelain, 218 p. Un Avant-propos et six chapitres : Des mœurs, Des instructions, Des amusements, Des voyages, Avis aux Pères, Avis au Précepteurs.

15. La Haye V. Vaillant et Prévost, 2 vol.; dédié à la Princesse de Galles, écrit pour le baron de Stain.

16. Références données plus haut note 2 .

17. Voir ce qu'il dit dans la préface. Voir aussi Traité, p. 268, et « Discours libre sur l'instruction », p. 6-11. Dans ce dernier texte, il remarque qu' " après huit ans d'études » au Collège, l'élève parle "plus mal le Latin que les plus vils esclaves ne le parlaient à Rome »; il considère l'apprentissage traditionnel du latin comme un archaïsme néfaste, au même titre que l'apprentissage par cœur de la grammaire, et voudrait voir utilisées «les voies abrégées ». Dans les Nouvelles maximes (ch. II, «Qu'il suffit de passer pour savant», p. 101), Crousaz souligne ironiquement que le latin en tant que «matière principale de l'instruction » est de l'intérêt du précepteur, pour qui rien n'est plus commode que d'occuper l'élève en lui donnant un long thème.

18. « Discours libres... », p. 15.

19. Traité, t. 1, p. 276.

20. Avril 1727, vol. 81, p. 443.

21. Voir le «Discours de la pédanterie" déjà cité, mais aussi les «effets pernicieux de la pédanterie » dans la Morale (t. 1, p. 208-209).

22. Traité, t. I, p. 390.

23. La logique ou l'art de penser, III, ch. XVII, «Des lieux ou de la Méthode de trouver des arguments. Combien cette méthode est de peu d'usage» (i.e. de peu d'utilité) ; éd. L. Marin, Paris, Garnier-Flammarion («Champs »), 1970, p. 293.

24. Crousaz, Logique, t. 5, p. 409-410 (sur Google). Le jeune écolier qui sait nommer les lieux «ne saura ni faire la paraphrase de la lecture, ou en rapporter le précis, ni en discuter les preuves, et en peser la force, et faut-il s'en étonner? On l'accoutume à se détourner des idées déterminés, qui font et la force et la beauté d'un discours pour se dissiper dans des notions vagues. » De même, il faut comprendre avant de donner l'« analyse logique ». Voir aussi p. 414 l'allusion à une histoire perdue écrite en syllogismes dont parle Lucien.

25. Traité, t. I, p. 281. Les élèves ne doivent pas apprendre les discours qu'ils ont écrits, mais « des harangues de Tite-Live, des morceaux de celles de Cicéron». Cette remarque permet de comprendre un des intérêts pédagogiques des discours pris à Tite-Live : ils sont plus courts que ceux de Cicéron. Sur la place de Tite-Live dans l'éducation, voir aussi, ibid., p. 265.

26. Le professeur doit montrer dans un texte que la pensée «étend ce qui a précédé, qu'elle prépare à ce qui va suivre » (« Discours libres », Divers écrits, t. 1, p. 14).

27. Crousaz, « Discours libre... », p. 5.

\section{AUTEUR}

\section{JEAN-YVES VIALLETON}

Univ. Grenoble-Alpes, RARE - Rhétorique de l'Antiquité à la Révolution 